\title{
Maximizing Chemical and Textural Data with Minimal Sample Destruction: Computed Tomography, Wire Saws, and Electron Beams; 'Oh, my'.
}

\author{
Ellen J. Crapster-Pregont ${ }^{1,2}$ and Denton S. Ebel ${ }^{2,1}$. \\ 1. Lamont-Doherty Earth Observatory, Columbia University, Palisades, NY, 10964, USA. \\ 2. Dept. of Earth and Planetary Science, American Museum of Natural History, New York, NY, \\ 10024,USA.
}

Chondrites, undifferentiated meteorites, have bulk chemistries similar to solar and preserve the history of the protoplanetary disk in their inclusions. The rarest subsets of chondrites with highest scientific value are only available for research in small aliquots. Minimally destructive techniques from sample collection to detailed chemical analysis are important for detailed understanding of relationships between not only inclusions in chondritic meteorites but also the mineral phases within each inclusion. A combination of non-destructive techniques through the entire sequence from sample selection, preparation, and analysis maximizes scientific return while minimizing material loss.

A 3-dimensional (3D) map of the sample preserves material by guiding future cuts and allowing predetermination of sample suitability for the study [1]. Computed tomography (CT) scanners utilize high-powered x-rays to create 3D density maps of a variety of samples. Obtainable resolution depends on sample size, or distance from source, and size of focal spot; e.g. resolution for a sample $5 \times 5 \times 20 \mathrm{~mm}$ is $\sim 4 \mu \mathrm{m} /$ voxel on the AMNH GE VtomeX-S (Fig. 1A and B). Resolution limits the types of analyses that can be conducted. Lower resolution allows virtual isolation (segmentation) and quantification of materials with significantly different densities (e.g. metals vs. silicates or chondrules vs. matrix) while higher resolution studies can differentiate different silicate and oxide minerals [e.g., 2-5].

Typical diamond studded rock-cutting blades lose a $>100 \mu \mathrm{m}$ thick slice of material. Use of a 20, 30 or $50 \mu \mathrm{m}$ tungsten (W) wire saw (Princeton Instruments) minimizes the thickness of material lost. This effectively minimizes sample loss and maximizes the number of surfaces that can be analyzed, a method called 'serial sectioning' (Fig. 1C ps1B and ps2A). Sub-400 $\mu \mathrm{m}$ diameter objects often are sectioned in more than two adjacent sets of surfaces [e.g., 2].

The Cameca SX100 electron microprobe (EMP) at AMNH allows use of two types of spectrometers: wavelength dispersive and energy dispersive, to create pixel-by-pixel element intensity maps (Fig. 1D). We convert these intensities to oxide wt $\%$ using similarly mapped standards that are also used to determine background intensity with minimal instrument time. Red-green-blue (RGB) composites of sets of three elements allow differentiation between types of inclusions and minerals in meteorites over large, region maps $(>1 \mu \mathrm{m} / \mathrm{pixel})$ or individual inclusions $(1 \mu \mathrm{m} / \mathrm{pixel})(\mathrm{Fig}$. 1D and E, inclusions outlined in white). Customized software evaluates each inclusion pixel-by-pixel using element intensity maps and combinations of ratios and cation formulas. A phase map is produced with each pixel assigned a false color indicating mineralogy determined by element intensities (Fig. 1F). Bulk chemistry, mineralogy, modal abundance, texture, and area are quantifiable from either region or individual inclusion maps (Fig. 1G). Unlike previous studies [e.g. 6-8] using 2D optical or electron beam techniques, this method emphasizes high-efficiency collection of quality data on large areas or numbers 
or inclusions, including 3D information (inclusion sizes and spatial relationships), and minimizing the time required for sample preparation, calibration, data acquisition, and image analysis.

Once characterized chemically, mineralogically, and texturally using this minimally destructive protocol, we choose inclusions, points, or areas for further, more destructive analyses (Fig. 1H; electron backscattered diffraction, EBSD; secondary ion mass spectrometry, SIMS; laser ablation inductively coupled plasma mass spectrometry, LA-ICP-MS; and focused ion beam liftout for transmission electron microscopy, FIB-TEM). Each of these techniques requires sample consumption to produce data.

Although there are many areas for improvement, our techniques provide examples of how each step in the analysis process can be optimized with the value of the meteorite and the resulting data in mind. When combined these techniques reduce the amount of material lost and maximize the information that can be obtained from a single meteorite sample. The results can systematically guide more destructive studies and preserve the initial chemical, mineralogical, petrological, and textural context in the element intensity maps and CT scans. By using a series of instruments, visualizations, and software protocols it is possible to begin to better understand the complexity of the protoplanetary disk, and planet formation processes preserved in meteorites with maximum conservation of these precious samples [9].

\section{References:}

[1] DS Ebel et al., Microscopy and Microanalysis 19, Supp. S2 (2013), p. 632-633.

[2] DS Ebel et al., Meteoritics \& Planetary Science 43, (2008), p. 1725-1740.

[3] JM Friedrich and ML Rivers, Geochimica et Cosmochimica Acta 116 (2013), p.63-70.

[4] SS Russell and L Howard, Geochimica et Cosmochimica Acta 116 (2013), p. 52-62.

[5] A Tsuchiyama et al., Geochimica et Cosmochimica Acta 116 (2013), p. 5-16.

[6] V De Andrade et al., Journal of Metamorphic Geology 24 (2006), p. 655-668.

[7] D Pret et al., American Mineralogist 95 (2010), p. 1379-1388.

[8] R Cossio and A Borghi, Computers and Geosciences 24 (1998), p. 805-814.

[9] This research is supported by NASA Cosmochemistry grant NNX10AI42G (DSE) and the National Science Foundation Graduate Research Fellowship Program grant DGE-11-44155 (ECP).

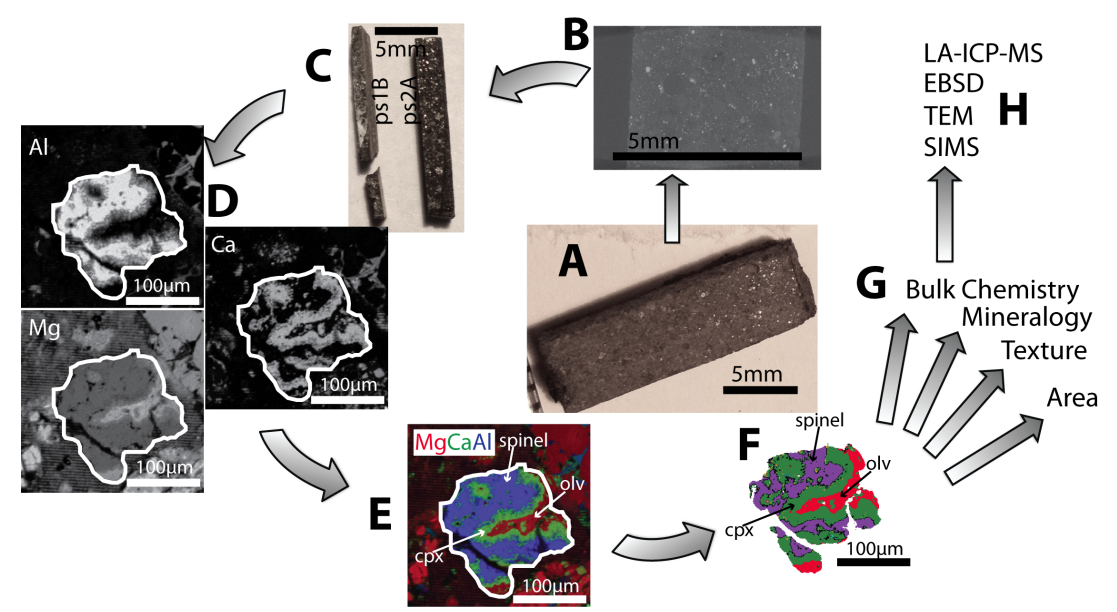

Figure 1: Preparation and analysis steps. (A) Photo of Moss (CO3.6) AMNH \#5185; (B) single CT slice, high density is whitest; (C) post-wire saw sections; (D) EMP element intensity maps with inclusion outlined; (E) RGB composite, note ease of distinguishing inclusion; (F) false color mineral map output: purple-spinel, red-olivine (olv), green-clinopyroxene (cpx); (G) quantitative data produced; (H) further destructive techniques possible using a high level of prior contextual knowledge (A-G). 